\title{
ON THE REALITY OF ZEROS OF BESSEL FUNCTIONS
}

\section{ABRAHAM HILLMAN}

We shall present some observations on the reality of zeros of Bessel functions of real order, that is, functions satisfying the differential equation

$$
z^{2} \frac{d^{2} y}{d z^{2}}+z \frac{d y}{d z}+\left(z^{2}-\nu^{2}\right) y=0
$$

with $\nu$ real. The two linearly independent solutions $J_{\nu}(z)$ and $Y_{\nu}(z)$ may be defined by

$$
J_{\nu}(z)=\left(\frac{z}{2}\right)^{\nu} \sum_{r=0}^{\infty} \frac{(-1)^{r}(z / 2)^{2 r}}{r ! \Gamma(\nu+r+1)}
$$

and

$$
\begin{aligned}
& Y_{\nu}(z)=\frac{J_{\nu}(z) \cos \nu \pi-J_{-\nu}(z)}{\sin \nu \pi} \text { for } \nu \text { not an integer, } \\
& Y_{n}(z)=\lim _{\nu \rightarrow n} Y_{\nu}(z) \text { for integers } n .
\end{aligned}
$$

$J_{\nu}$ and $Y_{\nu}$ are in general many-valued functions of $z$. If in (2) we replace $z$ by the positive real variable $x$ and use the principal value of $(x / 2)^{\nu}$, a real valued function, $J_{\nu}(x)$, is obtained. Substituting $J_{\nu}(x)$ for $J_{\nu}(z)$ in (3) gives a real function $Y_{\nu}(x)$.

All branches of any Bessel function, $B(z)$, can be obtained by analytic continuation of a function

$$
B(x)=(a+i b) J_{\nu}(x)+(h+i k) Y_{v}(x),
$$

where $a, b, h$, and $k$ are real numbers. In particular let $B(x, m)$ stand for the result of continuing $B(x)$ through an angle of $m \pi$ along a circle with center at the origin. Restricting $m$ to be an integer, it can be shown ${ }^{1}$ that

$$
\begin{aligned}
B(x, m)= & {\left[(a C-b S-2 k S \cot \nu \pi) J_{\nu}(x)+(h C+k S) Y_{\nu}(x)\right] } \\
& +i\left[(b C+a S+2 h S \cot \nu \pi) J_{\nu}(x)+(k C-h S) Y_{\nu}(x)\right]
\end{aligned}
$$

where $C=\cos m \nu \pi$ and $S=\sin m \nu \pi$. Each real (positive or negative) zero on any branch of the analytic function $B(z)$ is a zero of $B(x, m)$

Presented to the Society, April 17, 1948; received by the editors March 8, 1948 .

${ }^{1}$ See G. N. Watson, A treatise on the theory of Bessel functions. p. 75. 
for proper choice of $m$. Equating the real and imaginary parts of $B(x, m)$ to zero (here we use the hypothesis that $\nu$ is real), gives

$$
\begin{aligned}
& (a C-b S-2 k S \cot \nu \pi) J_{\nu}(x)+(h C+k S) Y_{\nu}(x)=0, \\
& (b C+a S+2 h S \cot \nu \pi) J_{\nu}(x)+(k C-h S) Y_{\nu}(x)=0 .
\end{aligned}
$$

$J_{\nu}(x)$ and $Y_{\nu}(x)$ are never simultaneously zero since they are linearly independent solutions of equation (1). Hence a necessary condition for $B(x, m)$ to have zeros is that

$$
\begin{aligned}
\Delta \equiv & (a C-b S-2 k S \cot \nu \pi)(k C-h S) \\
& -(b C+a S+2 h S \cot \nu \pi)(h C+k S)
\end{aligned}
$$

vanishes. This can be simplified to

(4) $\Delta \equiv[a k-b h] \cos 2 m \nu \pi-\left[a h+b k+\left(h^{2}+k^{2}\right) \cot \nu \pi\right] \sin 2 m \nu \pi$.

If $\Delta$ is equal to zero, $B(x, m)$ is a multiple of a real cylinder function. It is well known ${ }^{2}$ that the real cylinder functions have (positive) real zeros. This shows that $\Delta=0$ is also a sufficient condition for $B(x, m)$ to have zeros. (It is clear that there are two values of $m$ associated with each branch of $B(z)$, an even value for positive zeros and an odd one for negative zeros.)

Before going on to some interesting special cases it should be noted that every Bessel function has, on every branch and in both the left and the right half-planes, an infinite number of zeros which lie on or approach a horizontal straight line. (The line depends on the branch and the half-plane.) This is a consequence of the fact ${ }^{8}$ that

$$
J_{\nu}(z) \cos \alpha-Y_{\nu}(z) \sin \alpha
$$

where $\nu$ and $\alpha$ need not be real, has zeros whose real parts tend towards plus infinity and which are given by the asymptotic expansion

$$
\begin{aligned}
z_{s} \sim \beta & -\frac{u-1}{2^{3} \beta}-\frac{(u-1)(7 u-31)}{3 \cdot 2^{7} \beta^{3}}-\frac{(u-1)\left(83 u^{2}-982 u+3779\right)}{15 \cdot 2^{10} \beta^{5}} \\
& -\frac{(u-1)\left(6949 u^{3}-153855 u^{2}+1585743 u-6277237\right)}{105 \cdot 2^{15} \beta^{7}}-\cdots
\end{aligned}
$$

where $u=4 \nu^{2}$ and $\beta=(s+\nu / 2-1 / 4) \pi-\alpha$.

This implies that the condition for the non-existence of positive or negative real zeros on a particular branch (that is, for a particular value of $m$ ) of a Bessel function is the condition for the existence of

2 Ibid. p. 481.

Ibid. pp. 505-506. 
non-real complex zeros in that half-plane on that branch. However a Bessel function can have both real and non-real zeros in the same half-plane on the same branch. For example, if $r$ is zero or a positive integer and if $\nu$ is between $-(2 r+1)$ and $-(2 r+2), J_{\nu}(z)$ has $4 r+2$ complex ${ }^{4}$ zeros as well or an infinite number of positive and negative real ${ }^{5}$ zeros on each branch.

Now let us take up some of the special cases. We assume that $a, b$, $h, k$ are not all zero, that is, that $B$ is not identically zero.

I. Let $B$ be a multiple of $J_{\nu}$. That is, let $h=k=0$. Then the $\Delta$ of (4) is zero for all values of $m$ and $\nu$. In other words, $J_{\nu}$ has positive and negative real zeros on every branch for all real values of $\nu$.

II. Let $\nu$ be an integer $n$. Then $\Delta$ becomes $a k-b h-2 m\left(h^{2}+k^{2}\right)$. We see that with the exception of multiples of $J_{\nu}$ (treated under I), Bessel functions of integral order have real zeros if and only if $(a k-b h) / 2\left(h^{2}+k^{2}\right)$ is an integer and then only on the branch for which $m=(a k-b h) / 2\left(h^{2}+k^{2}\right)$.

In particular $Y_{n}(z)$ has positive real zeros only on the branch for which $m=0$ and has no negative real zeros. Thus $Y_{n}(z)$ has non-real complex zeros in the left half-plane on all branches and in the right half-plane on all but one branch.

III. Let $B$ be a multiple of $Y_{\nu}$, that is, let $a=b=0$. Then $\Delta=0$ is equivalent to $\cot \nu \pi \sin 2 m \nu \pi=0$. This means that $Y_{\nu}$ always has positive real zeros on the branch for which $m=0$ and has other real zeros only if $\nu$ is rational but not an integer. In the latter case $Y_{\nu}$ has real zeros only on the branches for which $2 m \nu$ is an integer. In particular $Y_{n+1 / 2}$ where $n$ is an integer has positive and negative real zeros on all branches.

IV. Let $B$ be a multiple of a function $\lambda J_{\nu}+\mu Y_{\nu}$ with $\lambda$ and $\mu$ real, that is, let $a k-b h=0$. Then $B$ has positive and negative real zeros on all branches if $B$ is a multiple of $J_{\nu}$ or of $J_{\nu} \cos \nu \pi-Y_{\nu} \sin \nu \pi$. Otherwise $B$ has real zeros if and only if $2 m \nu$ is an integer but $\nu$ is not.

$\mathrm{V}$. If $2 m \nu$ is an integer but $\nu$ is not then $B$ has real zeros if and only if it is a multiple of a function $\lambda J_{\nu}+\mu Y_{\nu}$ with $\lambda$ and $\mu$ real.

VI. The condition on $a, b, h$, and $k$ for $\Delta$ to be zero for all values of $m$ and $\nu$ is that $h=k=0$. (VI is the converse of I.)

Computation Laboratory, National Bureau of Standards

\footnotetext{
4 Ibid. p. 483.

5 Ibid. p. 478.
} 